
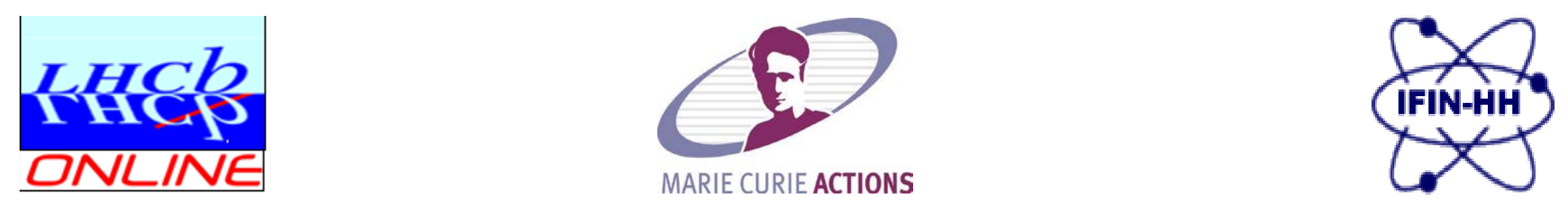

\title{
Data handling and transfer in the LHCb experiment
}

\section{RT NPSS Real Time 2007}

\author{
FNAL - $4^{\text {th }}$ May 2007
}

A.C. Smith, M. Frank, N. Neufeld, R. Stoica*

(CERN) 


\section{$\mathrm{LHCb}$ in a nutshell}

- One of the 4 major LHC experiments at CERN

- 2 proton beams colliding at $7 \mathrm{TeV}$ each

- Studies CP violation of b-mesons and other rare decays

- Single arm spectrometer

- 1.000 .000 channels to be read out at $40 \mathrm{MHz}$

- Foreseen to start at the end of 2007

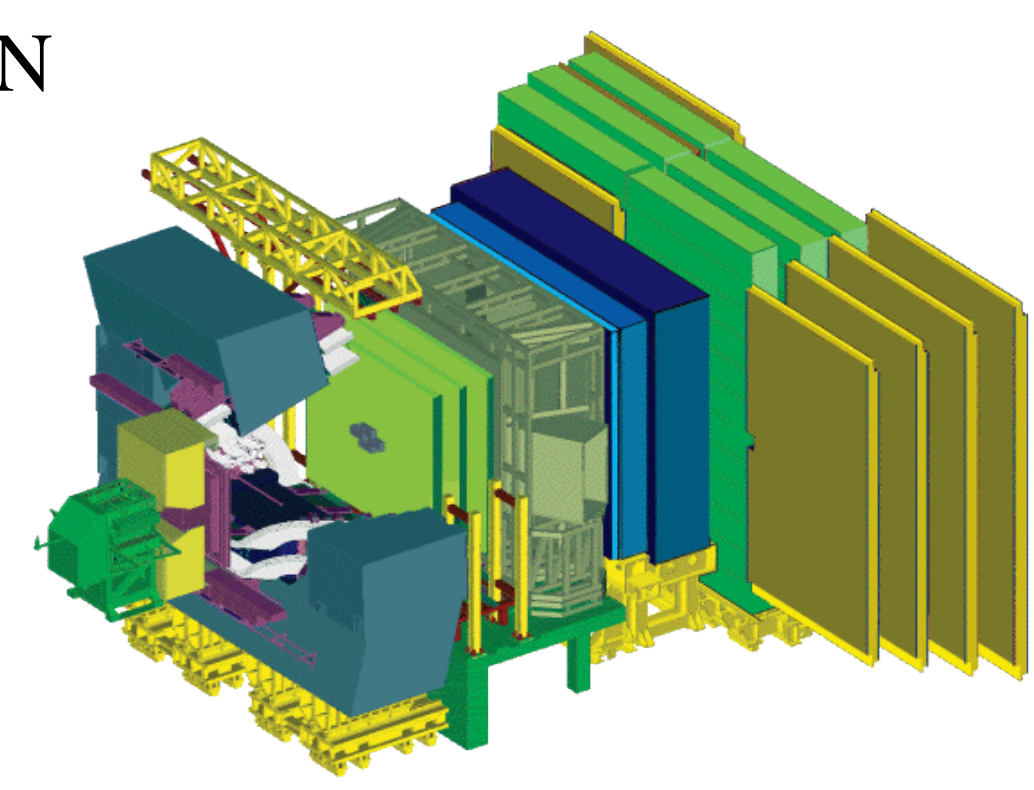




\section{DAQ System overview}

ONLINE

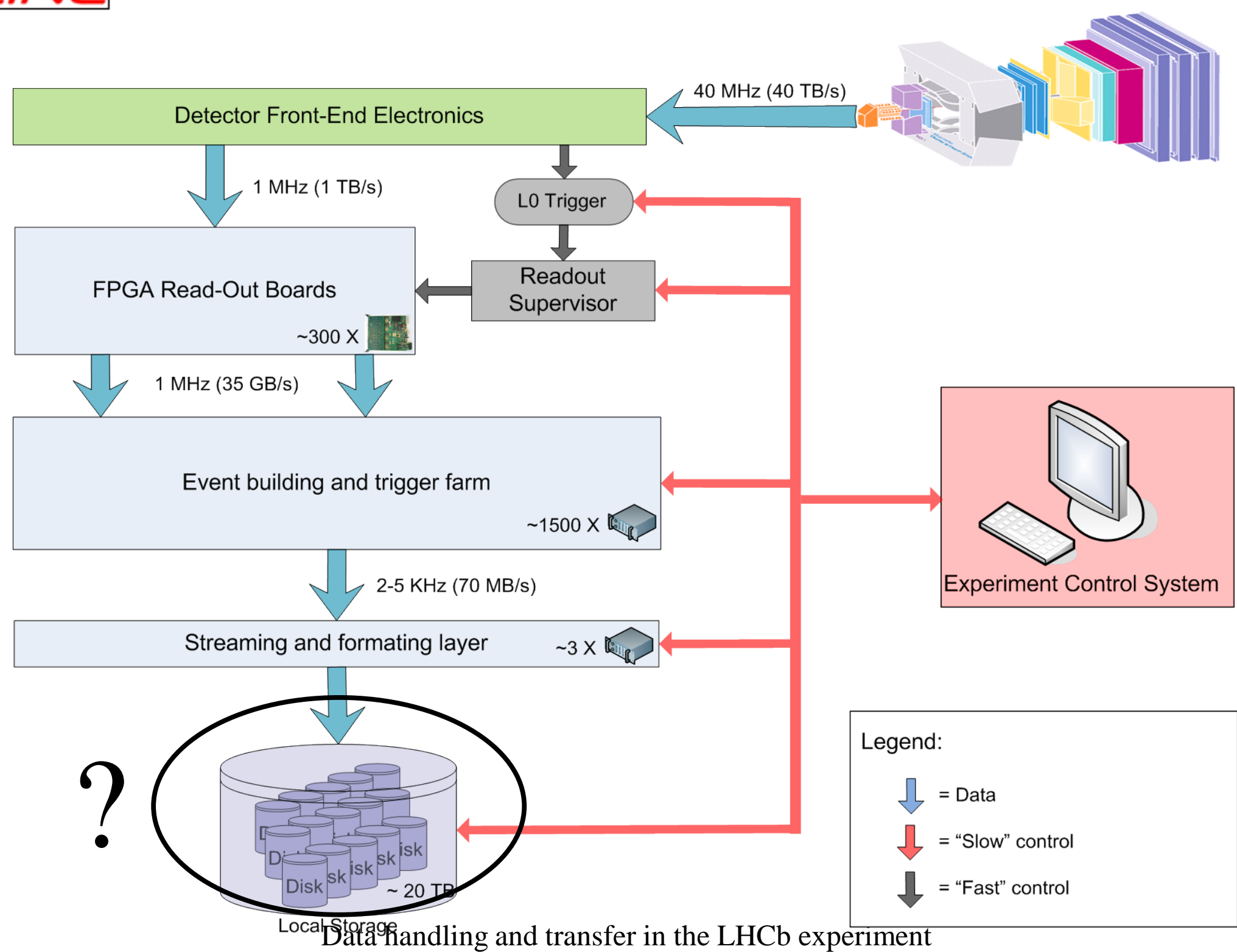




\section{File management requirements}

Functional requirements:

- Replicating files to the CERN Tape storage system (CASTOR)

- Creating appropriate entries in the GRID file catalogue (database that serves as a translation between the logical file name and its physical location)

- Managing metadata information and replicating it inside the LHCb Bookkeeping Database (database storing all information needed for all physics computing)

Operational requirements:

- Integration with the Experiment Control System

- Error recovery, redundancy

- Interface to external Online applications

- Automated and flexible management 


\section{File management implementation overview}

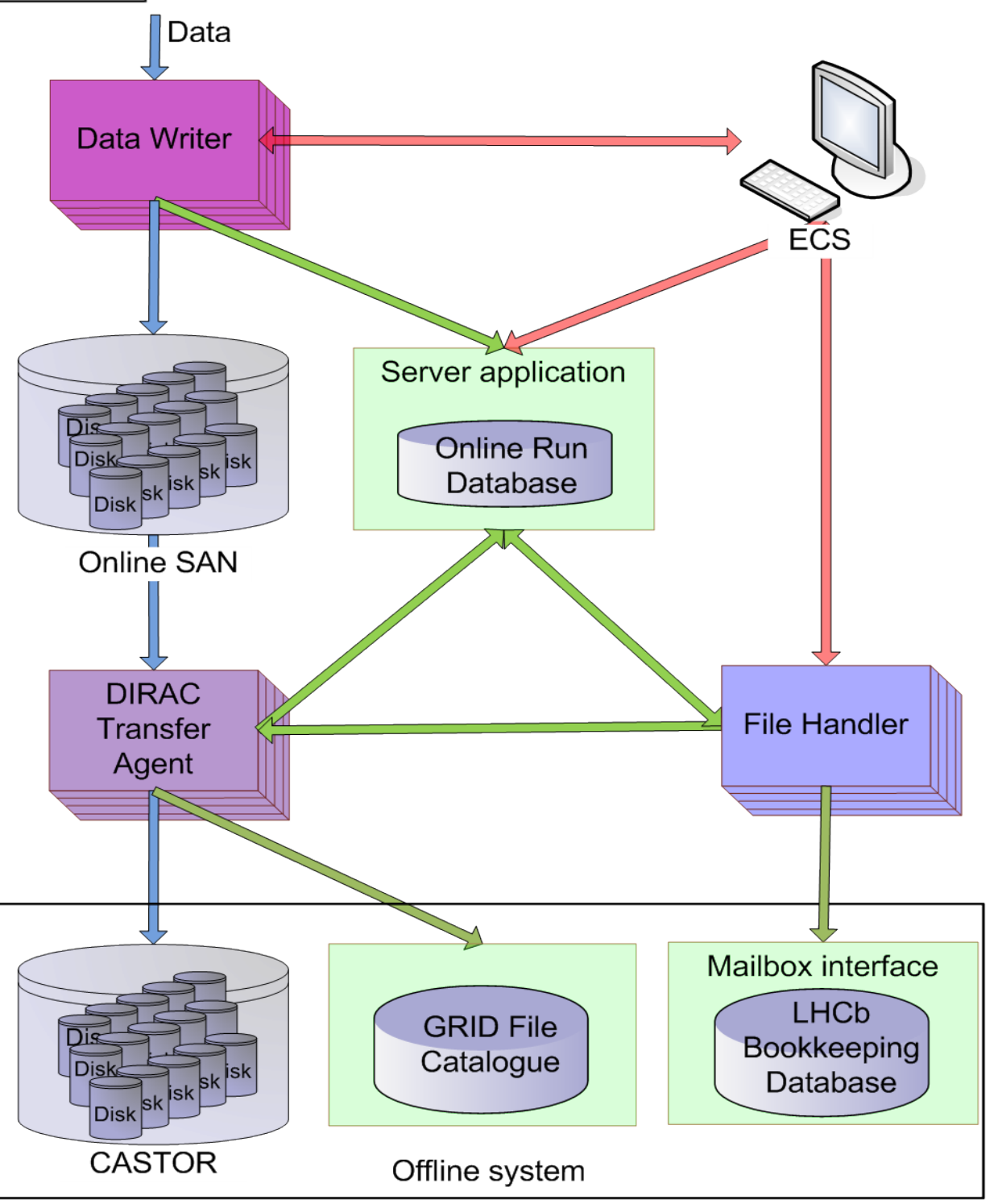

ECS = Experiment Control System

DIRAC = Distributed Infrastructure with Remote Agent Control

CASTOR = CERN Advanced STORage Manager

\section{Legend: \\ ¿ Data flow \\ ] Control communication \\ File handling communication}




\section{Online Run Database}

- Centralized point of file management.

- Simple but extensible SQL structure (not fixed yet!).

- Oracle database backend.

- Control and behaviour implemented using a server side Python application (using the cxOracle module)

- The management application is stateless, can be run in multiple instances for redundancy.

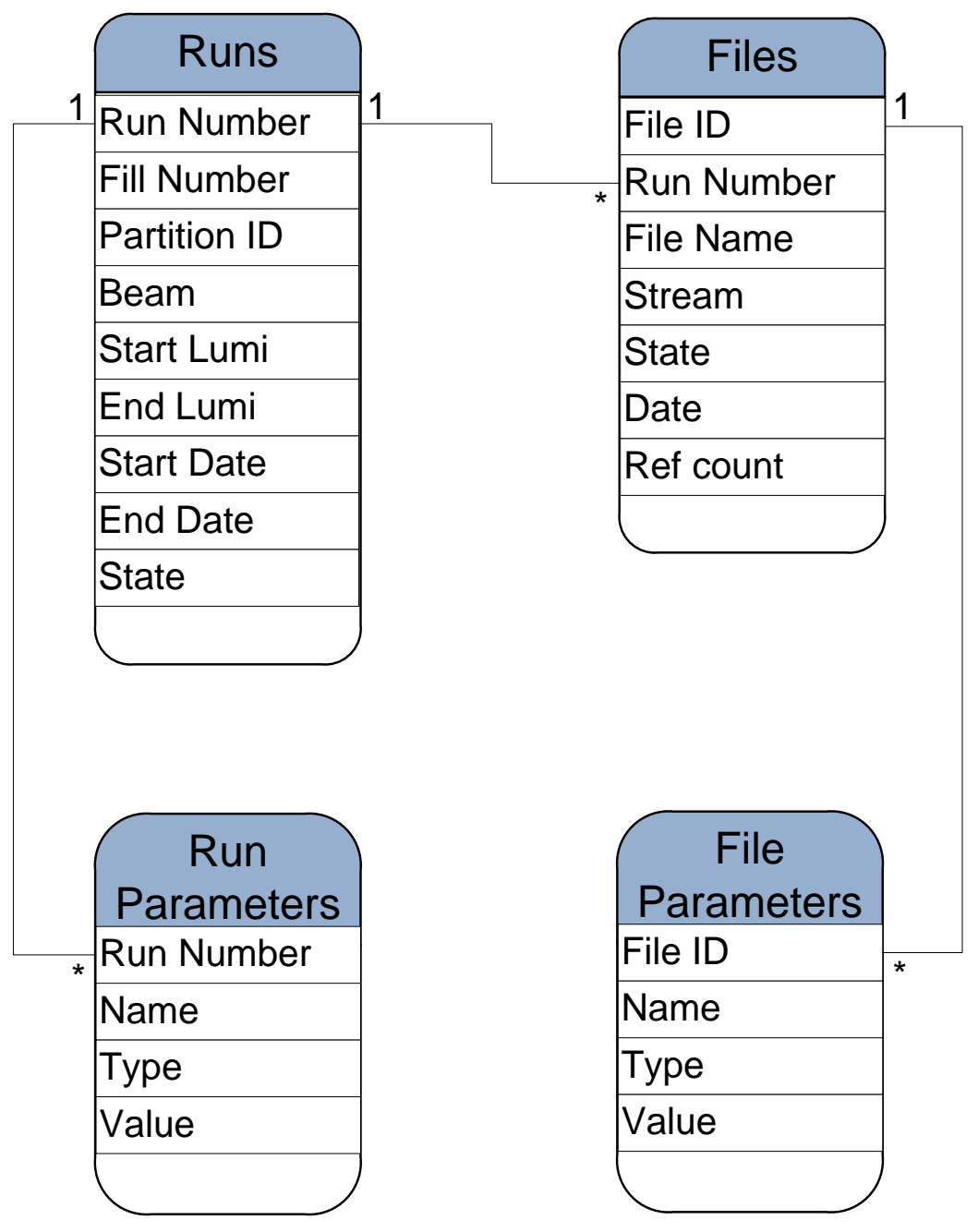




\section{File states inside DAQ System}

-Pseudo Petri net diagram models file behaviour

-Asynchronous transitions from one state to another

-File accesses are reference counted

-Timestamps are used for error recovery

-File migration is decoupled from file usage

-File deletion triggered by low space on the Online SAN

\section{Created \\ File in writing}

\section{Closed}

File closed

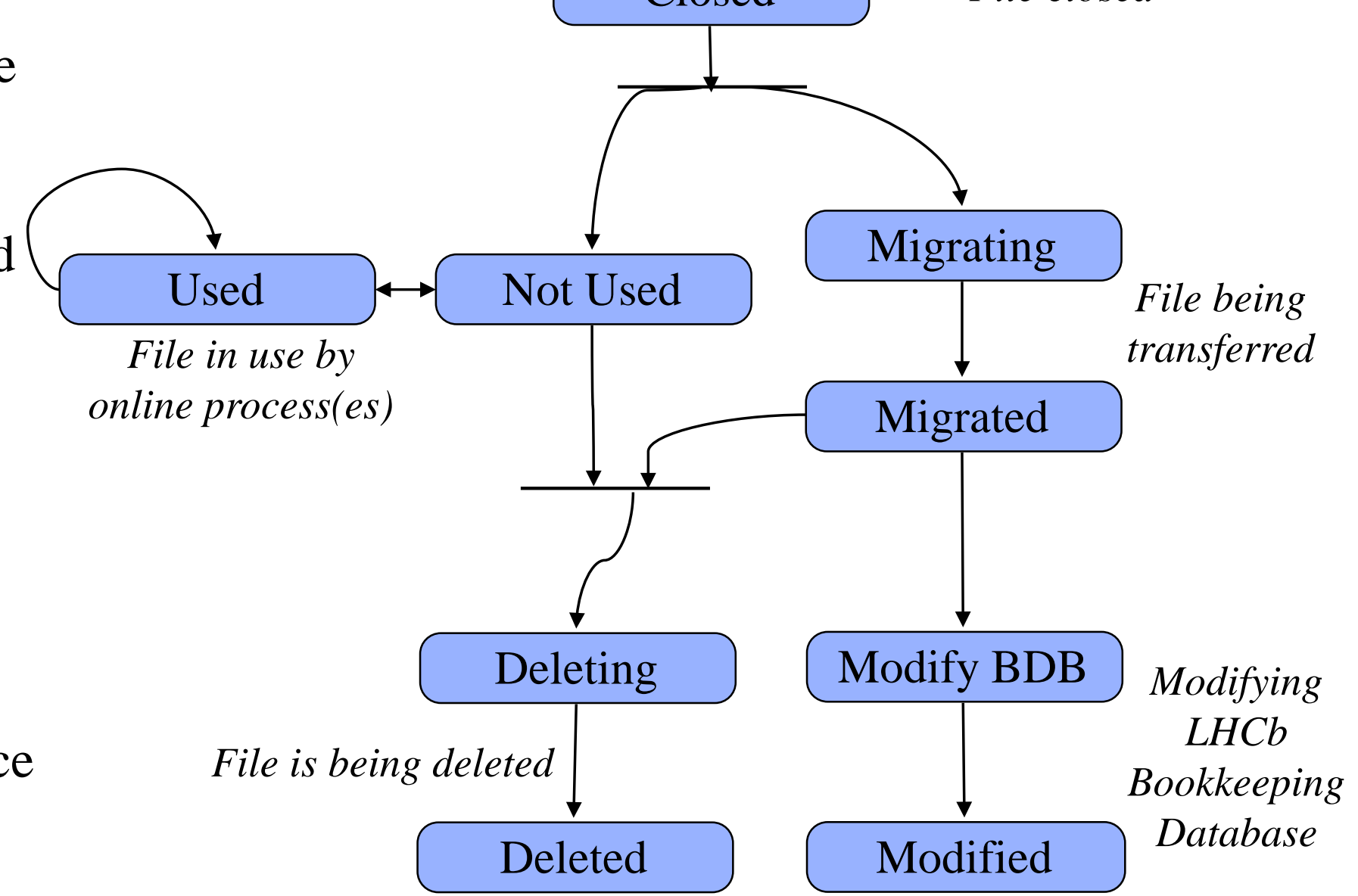




\section{Online Run Database interfaces}

ONLINE

Several possibilities for accessing/controlling the Online Run

Database:

- XMLRPC interface for the server controlling the actual Oracle database. Required for communication with the DIRAC software. Also useful for creating various scripts/applications (C/C++, Python, etc)

- DIM interface to the Experiment Control System (PVSS). DIM is exposed to Python as an ordinary C library.

- Oracle specific tools for performing "big" changes to the database (e.g. table modification, backup restoring).

- HTTP based interface that can be opened using a common web browser. Should be the most used tool for viewing the content of the database and for small administrative tasks. Supports access control. 


\section{File handler}

D INE

- Functionality:

- $\quad$ file copy to CASTOR (triggering file migration)

- $\quad$ updates the LHCb Bookkeeping DB

- $\quad$ file deletion

- $\quad$ error recovery

- $\quad$ Can be run in multiple instances on different machines if needed. The Online Database is the central point of synchronization (e.g. assures that the same file is not migrated simultaneously by two independent processes).

- Can run independently of the other online processes (independent of the Data Writer).

- $\quad$ Integrated with the ECS using DIM 


\section{WEB Management Tool}

ONLIAIE

File É dit $\underline{\text { View }}$ Go Bookmarks $\underline{T}$ ools $\underline{\text { Window }}$ Help

2 - 2 http://bonrac2/test.py

$\sim 2$ search

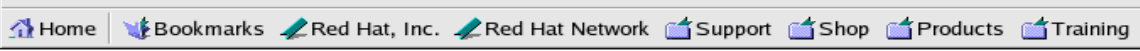

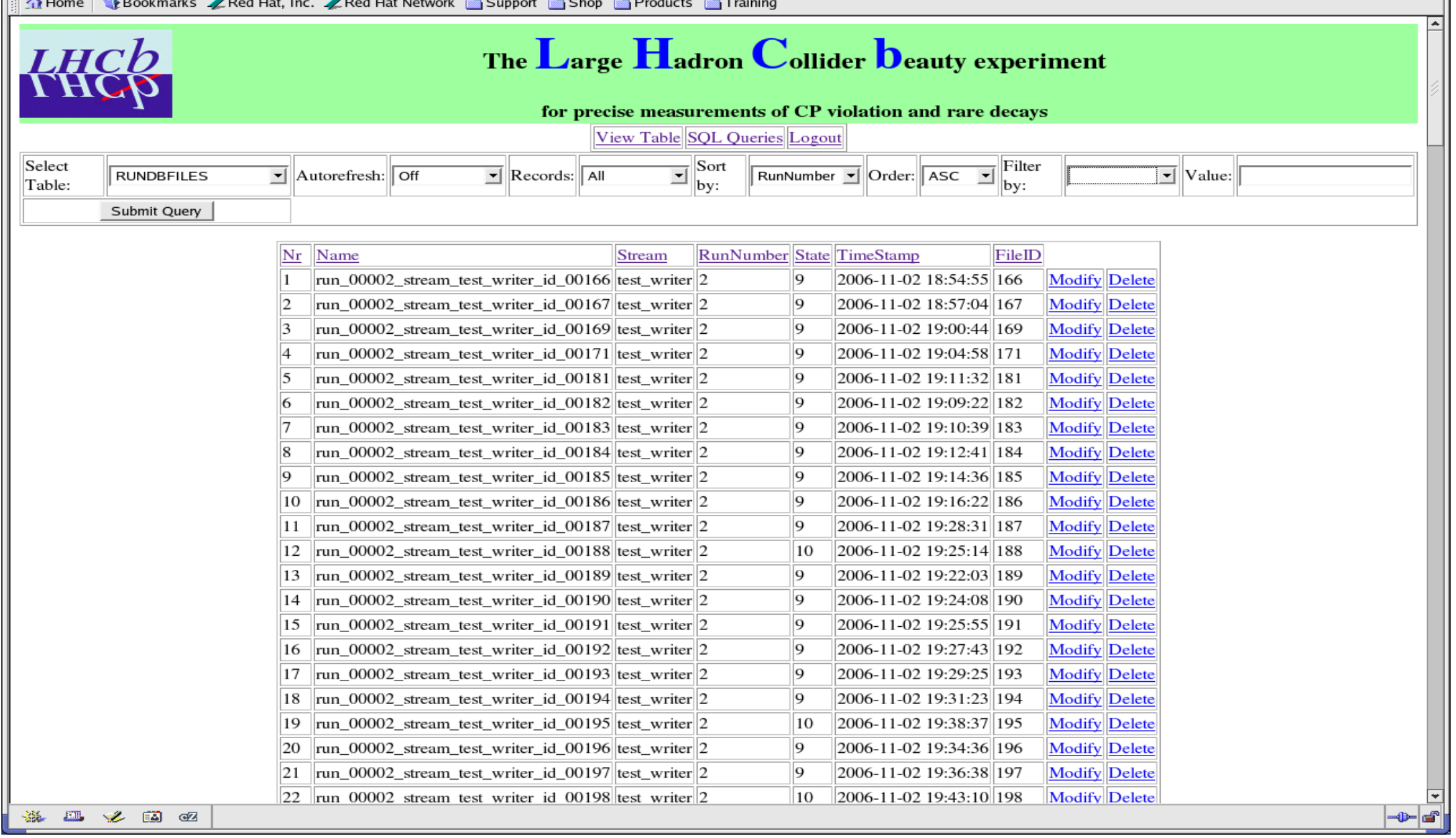




\section{Tests}

ONLINE

- Functionality: files generated and transferred to CASTOR. Complete software chain is working.

- Stability and error recovery:

- $\quad$ Tested behaviour and error recovery in case of simulated errors.

- Performance:

- Load simulated for the data handler and Online Run Database on a single machine. Maximum data handling was $10^{3}$ more than needed:

- files managed at a rate of $30 / \mathrm{s}$

- $\quad$ CPU utilisation $~ 80 \%$

- $\quad \mathrm{I} / \mathrm{O} \sim 4 \mathrm{MB} / \mathrm{s}$

- $\quad$ files entries: 8 million 


\section{Thank you!}

\section{ONLINE}

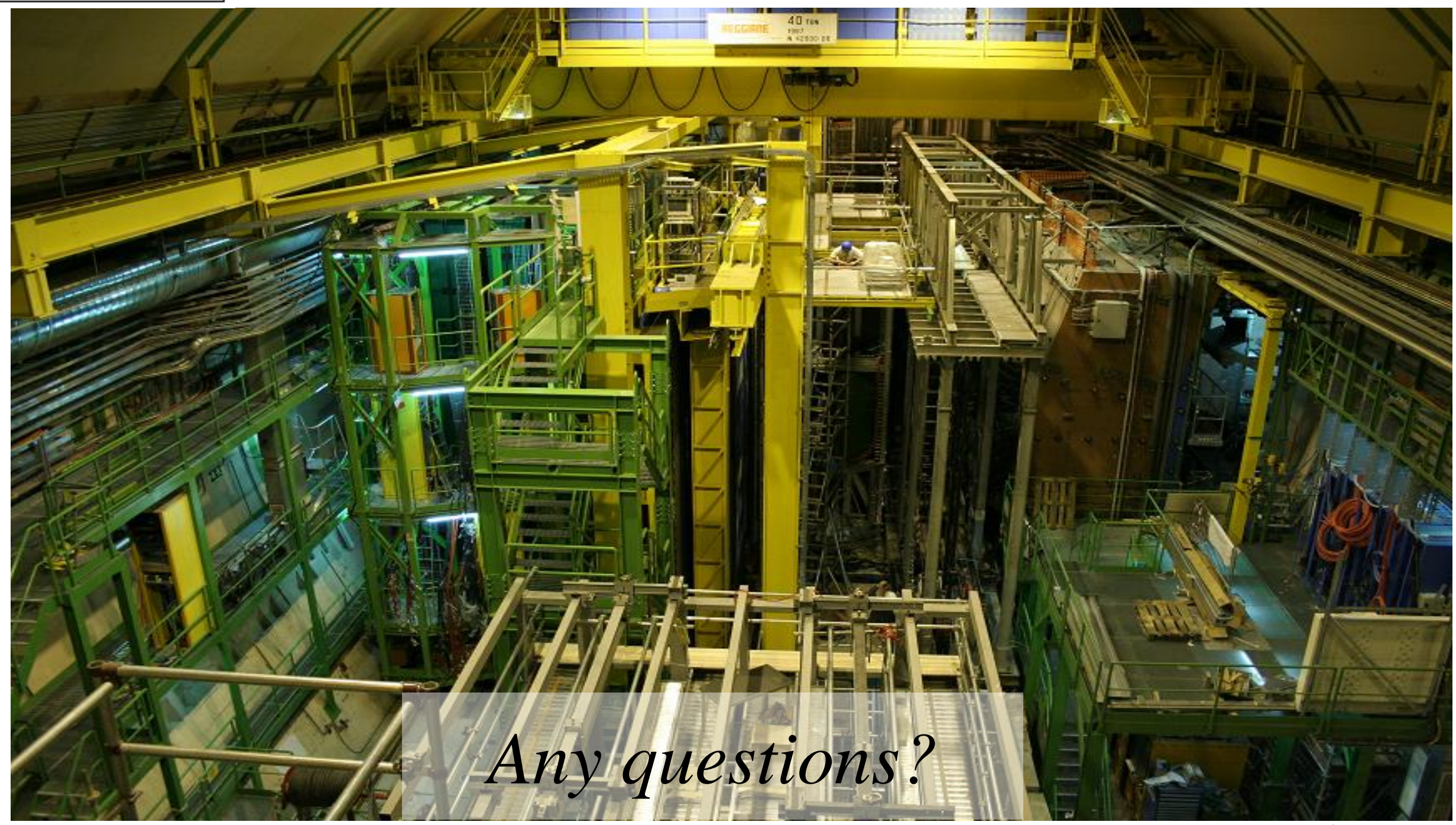

